\title{
Infrared response of charge-coupled devices
}

\author{
Matthias Loch, ${ }^{\mathrm{a}}$ Ralf Widenhorn, ${ }^{\mathrm{a}, \dagger}$ and Erik Bodegom ${ }^{\mathrm{a}, \mathrm{b}, \mathrm{t}}$ \\ ${ }^{\mathrm{a}}$ Department of Physics, Portland State University, Portland, OR 97207 \\ ${ }^{b}$ Department of Microelectronics, Delft University of Technology, 2628 CD Delft, Netherlands
}

\begin{abstract}
With a band gap of silicon of $1.1 \mathrm{eV}$, the largest wavelength that can excite electrons from the valence to the conduction band is roughly $1100 \mathrm{~nm}$. As a consequence, in, for instance, a charge-coupled device, the quantum efficiency (QE) for wavelengths larger than $1100 \mathrm{~nm}$ is assumed to be zero. We found that there is a response at those longer wavelengths and that the response decreases with increasing wavelength. The QE increases with increasing chip temperature which suggests a thermally activated process. Impurities in the silicon provide the energy levels in the band gap, from which electrons can be excited either thermally or by absorption of a photon. It is these impurities that contribute to the infrared response. We characterized the response at chip temperatures of $248 \mathrm{~K}$ to $293 \mathrm{~K}$ for wavelengths from $1200 \mathrm{~nm}$ to $1600 \mathrm{~nm}$ and calculated the activation energies at these wavelengths. We found that hot pixels, i.e., pixels with extraordinary high counts in a dark frame, tend to respond stronger to infrared light than normal pixels. This correlation gets stronger for longer wavelengths. It is argued that this response can be used for probing the impurities present in the silicon bulk of the sensors.
\end{abstract}

Keywords: CCD, infrared, impurities, activation energy

\section{INTRODUCTION}

The mode of action of a CCD imager is to convert photons hitting the imaging chip into electrons, which are stored and then read out. The imaging chip is divided into individual elements, the pixels. The size and number of the pixels defines the spatial resolution of the camera. To characterize the light sensitivity of a CCD camera the Quantum Efficiency (QE) is used. It is the ratio of output and input and thus, describes the ability to convert incoming photons into measurable electrons. Generally, it is assumed that the $\mathrm{QE}$ for wavelengths longer than $1100 \mathrm{~nm}$ reduces to zero. ${ }^{1-4}$

Hence, CCDs are not expected to respond in the near infrared range. At a first view this seems only logical. For silicon the band gap between valence and conduction band is about $1.12 \mathrm{eV}$ at room temperature. Hence, photons which carry less energy should not be able to excite electrons into the conduction band. Only light with wavelengths shorter than approximately $1100 \mathrm{~nm}$, will be detected.

In this paper, we will show experimental data with wavelengths from $1200 \mathrm{~nm}$ to $1600 \mathrm{~nm}$. The results will show a small but nevertheless measurable QE. The response is temperature dependent, suggesting a thermally activated process. For such a process impurity or defect levels in the band gap might provide the intermediate states required for the electrons to hop into the conduction band. Examples of such states are isolated vacancies, impurity atoms, and dislocations. A model will be presented to explain that process.

A special relationship was found between hot pixels, i.e., pixels that have an intrinsically much higher dark current, and their infrared response. Hot pixels are distributed randomly over the CCD sensor. They are caused by defects or impurities in the silicon chip. With increasing wavelength we found that hot pixels respond stronger to the infrared light than regular pixels.

\footnotetext{
${ }^{\dagger}$ Electronic mail: ralfw@pdx.edu

‡Electronic mail: bodegom@pdx.edu
} 


\section{MEASUREMENTS}

In this experiment a SBIG ST-8XE CCD (Santa Barbara Instrument Group) camera was studied. The sensor is Kodak KAF-1602E, a 1530 by 1020 pixel array with individual pixel size of 9 x 9 microns and a full well capacity of $\sim 50,000$ electrons. A water cooled thermoelectric element is used to set and stabilize the temperature to an accuracy of $\pm 0.1 \mathrm{~K}$ down to approximately $50 \mathrm{~K}$ below ambient. The average dark current at $273 \mathrm{~K}$ was given by approximately 1 electron/pixel/second.

Monochromatic light in this experiment was obtained by the use of a monochromator made by Optometrics, model MLM-2. It consists of a light source, a tungsten light bulb, a power supply, an iris and shutter, and an Optometrics MiniChrom monochromator. The monochromator is an in-line Fastie-Ebert monochromator. The light passes the iris, the slit, the monochromator and finally gets focussed by a lens onto the exit slit. On the way to the sensor three high pass filters cut out wavelengths smaller than $1200 \mathrm{~nm}$. Two of them are located at the entrance and exit slits of the monochromator. The third filter is attached directly in front of the aperture of the camera and minimizes stray light. For longer wavelengths $(\geq 1350 \mathrm{~nm})$ a band pass filter was placed between monochromator and the focussing lens. Those filters became necessary since the QE decreased significantly for longer wavelengths so that even photons at wavelengths of between $1200 \mathrm{~nm}$ and $1300 \mathrm{~nm}$ can significantly distort the results for the longer wavelengths. In particular, with the ruled gratings of the monochromator so-called Rowland ghosts typically appear due to periodic errors in the spacing of the ruled grooves. ${ }^{5}$ The five band pass filters have center wavelengths of $1350,1400,1450$, 1500, and $1580 \mathrm{~nm}$ with a FWHM of approximately 25nm (Lambda Research Optics).

To measure the $\mathrm{QE}$ of the system, absolute measurements of the incident irradiance are needed. A Germanium Detector Module (GDM-1) from Optometrics is used; it consists of a mounted germanium photocell, integral low-noise highspeed operational amplifier, aperture assembly and housing. It has a photosensitive area of $5 \mathrm{~mm}$ in diameter. The spectral range goes from $800 \mathrm{~nm}$ to $1800 \mathrm{~nm}$.

The results of the monochromator were compared with data obtained with a $1310 \mathrm{~nm}$ laser diode (a continuous light $10 \mathrm{~mW}$ AlGaAs laser diode with a stable, single transverse mode oscillation). Another laser diode used was an InGaAsP single transverse mode Fabry-Perot laser with an optical output power of $6 \mathrm{~mW}$ and a peak emitting wavelength of 1520 $\mathrm{nm}$ to $1580 \mathrm{~nm}$. The same cut-off filters as in the previous experiment were used in the setup with diode lasers as the IR source. For the InGaAsP laser an additional $1580 \mathrm{~nm}$ band pass filter was used, because it seemed some light off the central wavelength was generated by the diode.

For a given wavelength, ten temperatures were used from $248 \mathrm{~K}$ in increments of $5 \mathrm{~K}$ to $293 \mathrm{~K}$. For each temperature a waiting period of thirty minutes for each $5 \mathrm{~K}$ step guaranteed that the temperature was stabilized. The exception was for the InGaAsP laser, where in between $3 \mathrm{~K}$ steps, there was a two minute waiting time. All wavelengths within one data set are exposed for the same time. Each series is measured with the same experimental setup with either the monochromator and the light bulb or the laser diode as light source.

\section{RESULTS}

Figure 1 shows the results for the QE at different temperatures for wavelengths from 1220 to $1290 \mathrm{~nm}$. The data are corrected for dark current and contain only the signal due to the IR radiation. One sees immediately the reason that CCDs are not very useful in the NIR range above $1200 \mathrm{~nm}$ : the QE starts at $10^{-6}$ and drops to $10^{-7}$ at the higher end of this wavelength range. In the visible range the $\mathrm{QE}$ is close to one, or a difference between visible and infrared of seven orders of magnitude. These results explain why the experiment has to be done with special care. For every photon of visible light it is at least $10^{6}$ times easier to excite electrons into the conduction band. It is not possible to distinguish counts between visible and infrared light and one has to be sure that the incoming light is really infrared light. One can see that the QE decreases approximately exponentially with increasing wavelength. Most importantly, although one would not expect a response for wavelengths longer than $1100 \mathrm{~nm}$, one observes a wavelength and temperature dependent response. The uncertainty in the individual data is approximately $20 \%$. This might seem large, but in effect is mostly due to which part of the sensor is probed. An example of this can be seen in the data in Fig. 1. Alternating 
wavelengths were obtained on different days; due to a rearrangement of the setup, the part of the sensor illuminated was changed (only about 8,000 out of $1.5 \mathrm{M}$ pixels are illuminated in this setup). Hence, the data is specific to the part of the sensor illuminated. This is a possible indication that the localized defects play a role in the response. The model is thus one in which defects with energy levels in the band gap provide the mechanism to enable photons with energy less than the band gap to cause a response. Probing the IR response thus becomes a potential novel way to study defects in silicon.

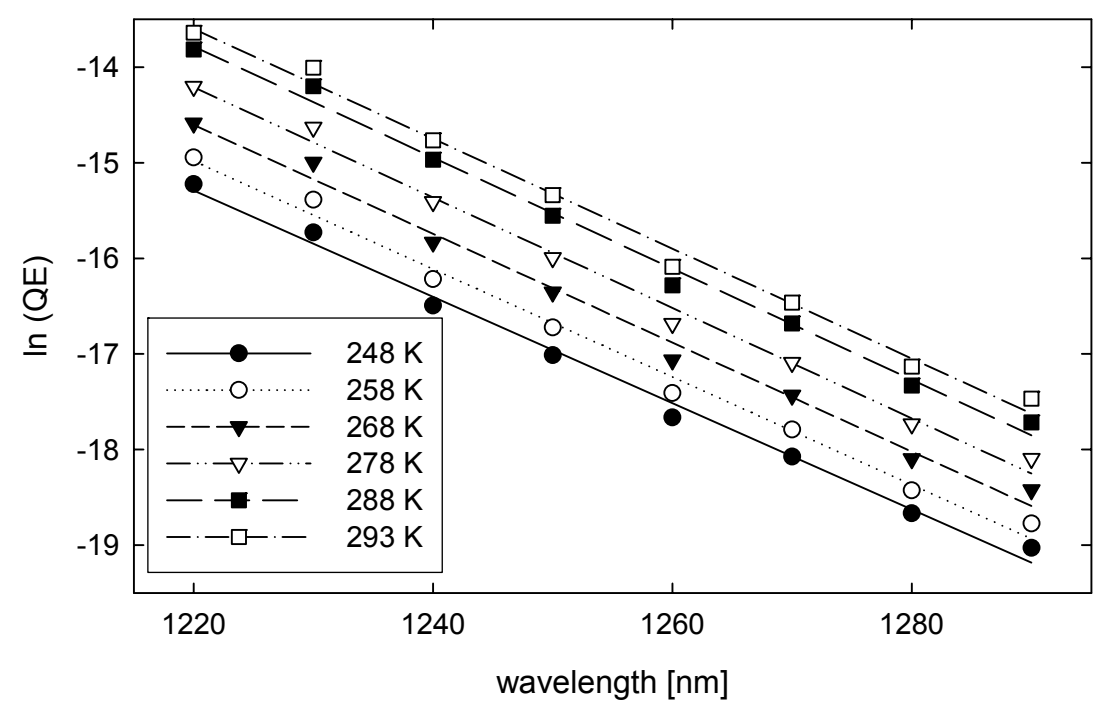

Figure 1. The natural logarithm of the quantum efficiency as a function of wavelength at various temperatures. A linear fit is drawn to guide the eye.

Figure 2 shows explicitly the temperature dependence at various wavelengths. It can be seen that for longer wavelengths the QE decreases significantly. The data for a given wavelength is done on the same part of the sensor and one notices that the data looks quite acceptable in the sense of the absence of scatter. These data lend credence to the explanation for the scatter in the data in Fig. 1, since the Fig. 2 data were obtained on one part of the sensor. It is important to realize that it is relatively easy for photons of $1220 \mathrm{~nm}$ to falsify measurements at a wavelength of $1290 \mathrm{~nm}$. To study wavelengths longer than $1300 \mathrm{~nm}$, the setup has to be modified from monochromator, light bulb, and high pass filters because the QE will be extremely low.

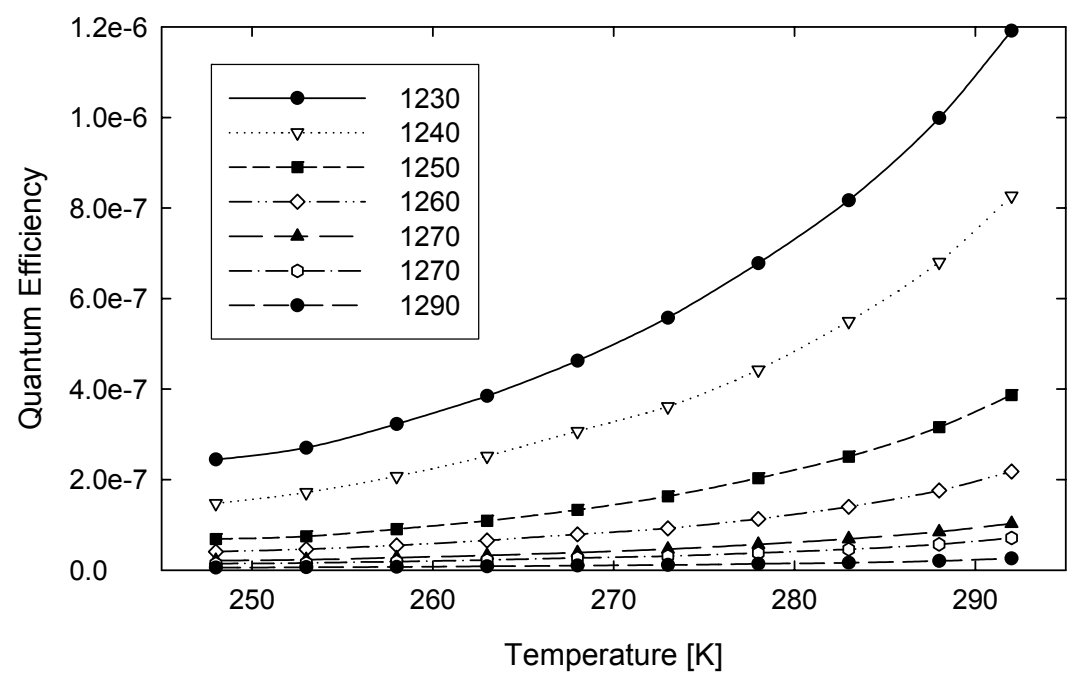

Figure 2. The dependence of the quantum efficiency on temperature for seven different wavelengths. 
Looking at the results of the $\mathrm{QE}$ versus temperature, it seems as if the $\mathrm{QE}$ increases exponentially with the temperature. Since the response to the infrared light is temperature dependent, we can consider the activation energy of this process. Looking at the Arrhenius plot for a given wavelength (Fig. 3), one sees that a reasonable fit to the data is obtained. The straight line fit through these points brings out that there is a slight curvature in the data. A possible explanation is that multiple processes with different activation energies play a role.

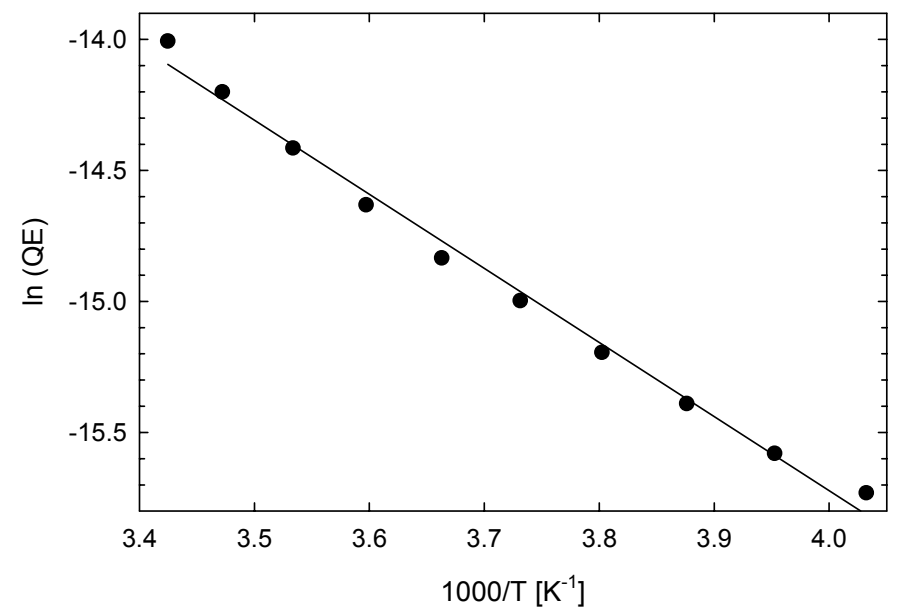

Figure 3. Arrhenius plot for $1230 \mathrm{~nm}$ : the natural logarithm of the quantum efficiency vs. temperature.

The IR-response, $y$, is approximately given by the following equation: $y=y_{0} \exp (-\Delta E / k T)$ with $k T$ being the product of Boltzmann's constant and the absolute temperature. The slope of the line in Fig. 3 gives the activation energy as: $\Delta E=238 \mathrm{meV}$.

The same procedure can be used for the other wavelengths. As one can see in Fig. 4, the slope and hence the activation energy, seems to decrease with increasing wavelength. That means that with longer wavelengths, and therefore smaller photon energy, the activation energy gets smaller, too. It is to be observed that this is surprising. If simple (near-band edge) impurities are the root cause, one would expect the activation energy to be the same for all wavelengths.

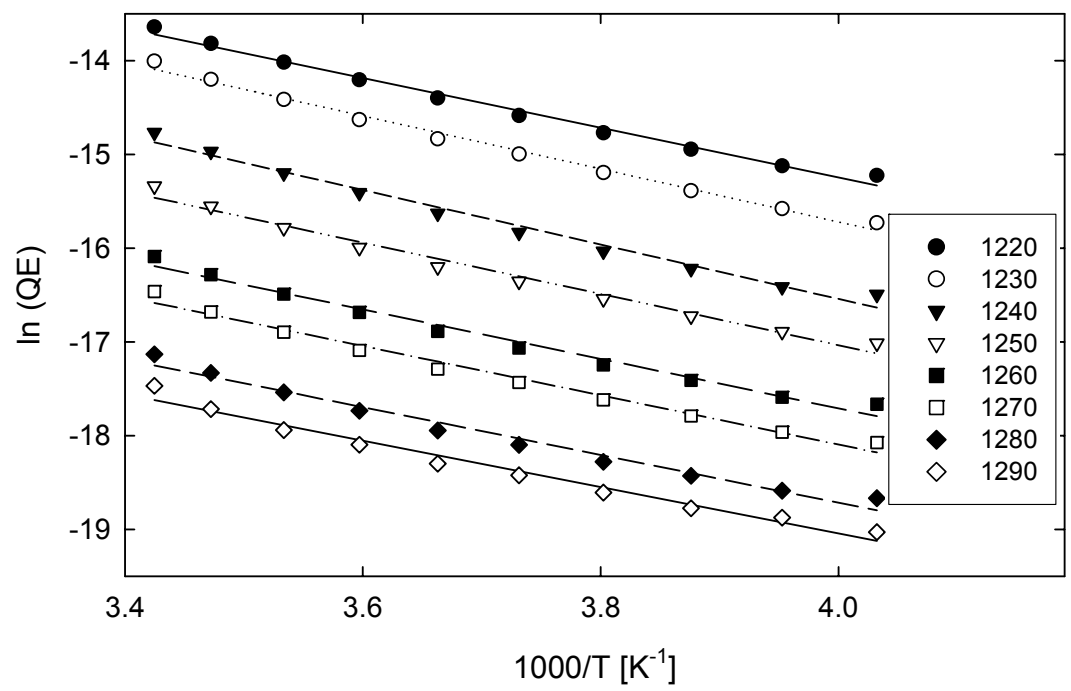

Figure 4. Arrhenius plots of natural logarithm of the quantum efficiency for wavelengths from $1220 \mathrm{~nm}$ to $1290 \mathrm{~nm}$.

In order to compare data sets for the various wavelengths and therefore different exposure times, one has to be sure that the exposure time has no influence on the activation energy. We have taken data for 30, 60, and 300 seconds exposure 
time; the count rate or infrared response, stayed the same and the activation energies, within the noise margins, showed no change.

As noted before, for longer wavelengths besides the monochromator, light bulb, and high pass filers, the band pass filters were used. The activation energies were calculated as well for the wavelengths defined by the band pass filters (see Fig. 5). For data taken at different times under similar conditions, the activation energies varied. One has to note that the signals are extremely weak and therefore the analysis of the data is sensitive to all sorts of interferences (e.g., small changes in the light emission properties of the light bulb, vibrations of the setup, etc.). Hence we used laser diodes.

The use of diodes has two main advantages. First of all, a laser diode insures that much less light, away from a single, narrow range of wavelengths, will be present. Second, one can achieve a higher intensity at these longer wavelengths. In order then to compare with the data at shorter wavelengths, one has to be sure that changes in intensity do not change the activation energy. Therefore the intensity of the laser at $1315 \mathrm{~nm}$ was varied by a factor of ten and the activation energies at the different power levels were calculated. The activation energies at those different intensities were within the experimental errors at that wavelength (4\%).

Figure 5 shows a summary of the data obtained using the light bulb and the laser diodes. The measured activation energies decrease from $\sim 230 \mathrm{meV}$ to $\sim 190 \mathrm{meV}$ beyond about $1450 \mathrm{~nm}$. The laser diode data at $1310 \mathrm{~nm}$ and the light bulb data are in agreement. The InGaAsP laser diode was used with the band pass filter at $1570 \mathrm{~nm}$. The spread in the activation energy (see Fig. 5) for different data sets was much larger than for those with the light bulb. A possible reason for this is that with the light bulb, extraneous, shorter wavelengths passed by the monochromator led to incorrect measurements. In effect we measured the activation energy associated with those extraneous and shorter wavelengths. For the spread in activation energy obtained with the diode, no pattern is discernible. Many reasons can be identified. First of all, the QE is extremely low. Although for most normal circumstances the diode provides a constant output, it does not. It fluctuates both in the dominant wavelength and in direction, thus a spread might occur in the measured activation energy. Due to the variable speckle pattern generated by the setup, the intensity can differ significantly from one pixel to another. These fluctuations provide varying intensities at a pixel and increase the uncertainties associated with the measurements. For shorter wavelengths the overall non-uniformity was not significant. Potentially, at $1570 \mathrm{~nm}$ other impurities in the chip could be affected and therefore the intensity variations might become important. The fluctuations in intensity and wavelength, the non-uniform illumination, the smaller area of the illumination, the weaker signal, and the shorter stabilization time of the temperature (two minutes after each $3 \mathrm{~K}$ change) all contribute to the spread in activation energy and the increased uncertainties at longer wavelengths.

As mentioned previously the data from the light bulb beyond $1310 \mathrm{~nm}$ is suspect, quite possibly due to the phenomenon of Rowland ghosts. One observes clearly the much larger uncertainties at the longer wavelengths. To explore the variations at these wavelengths, a more detailed study was made of the response of individual pixels.

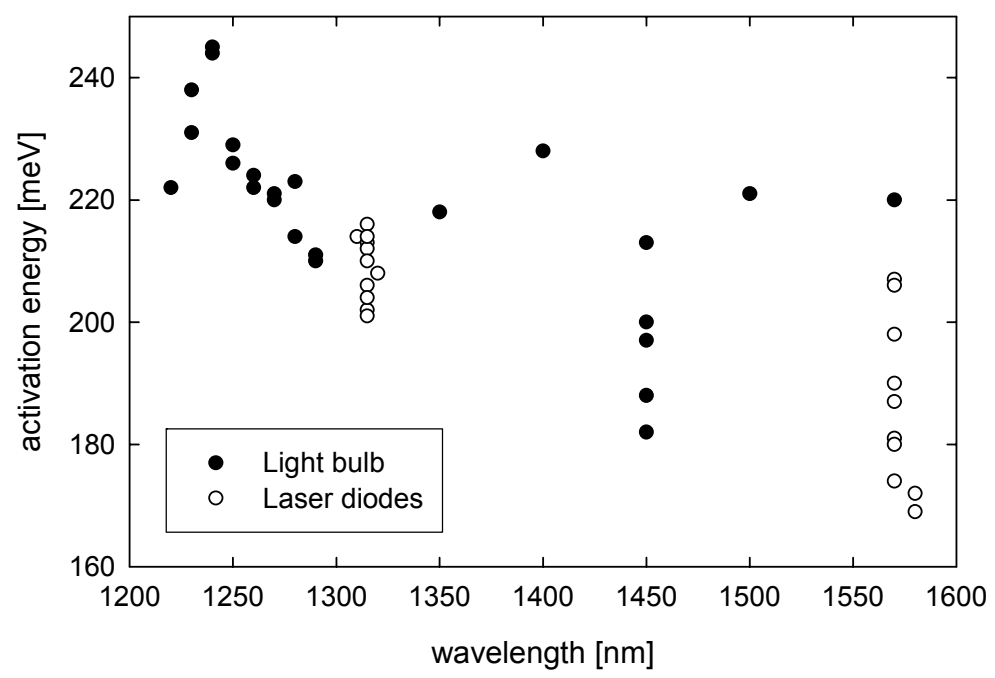

Figure 5. Activation energy as a function of wavelength (data obtained with laser diodes are marked with the open circles). 
There is a distinct relationship between hot pixels and infrared light. This relationship is more pronounced for longer wavelengths. We present data for a wavelength of $1580 \mathrm{~nm}$. Hot pixels can be seen in each dark-frame. Defects are responsible for the increased signal in these pixels.

Figure 6 shows the dark count for individual pixels on the $\mathrm{x}$-axis and the IR response of the respective pixel under illumination on the y-axis. This data set was taken at a temperature of $252 \mathrm{~K}$ for an exposure of 4 hours. 1365 pixels were included in the figure.

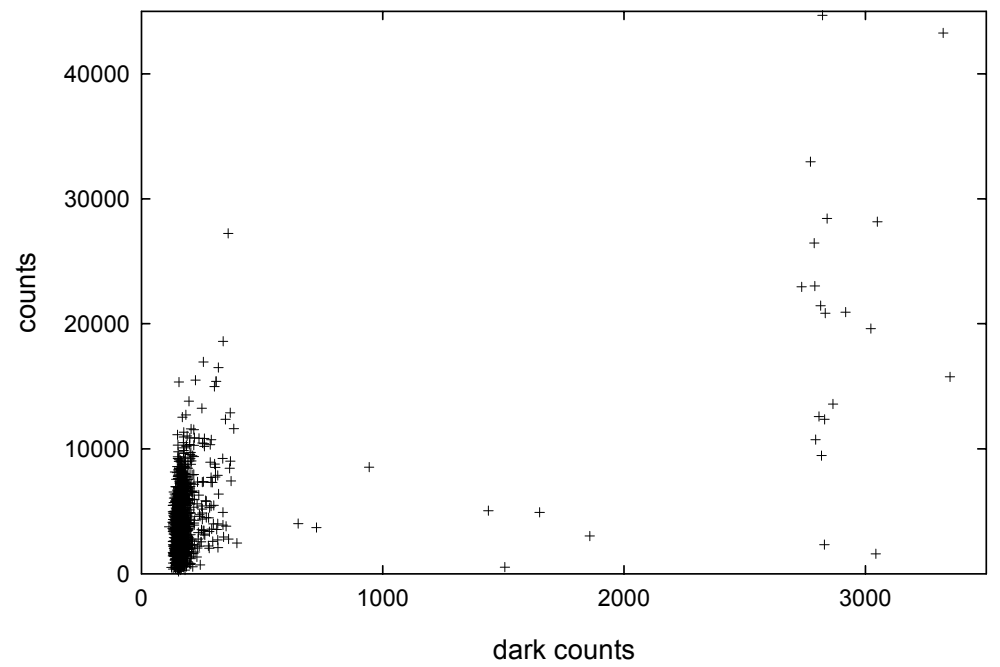

Figure 6. Counts in individual pixels under illumination of $1580 \mathrm{~nm}$ versus the dark count of those pixels at $252 \mathrm{~K}$.

One can see that there are two distinct and one diffuse regions. The first distinct region has a dark current of about 150 to 350 and the second distinct region has a dark current of about 3000 . The diffuse region contains some fifteen pixels and has no discernable pattern. All of the pixels in the second region are hot pixels; all in the first one are normal pixels. Furthermore, one can see that the response of hot pixels is larger than that of the normal pixels. Of the twelve pixels with counts over 20,000,11 pixels are hot pixels. No other pixel on the whole chip did have a response that high. That means that the highest response is found in the area of the illumination and additionally that these pixels, except for one, are hot pixels.

We also measured the average quantum efficiency at $1570 \mathrm{~nm}$ (see Fig. 7). The QE is much smaller than in the $1200 \mathrm{~nm}$ regime; in fact, it is six orders of magnitude smaller. Combined with Fig. 6, one can infer that the QE for hot pixels must be larger than for normal dark count pixels. The QE of hot pixels is about ten times larger than the average QE. Assuming that defects in individual hot pixels are the cause of the IR response, one can calculate the product of the cross section $(\sigma)$ and the number $(N)$ of active defects present in the pixel. $N \sigma$ equals the number of electrons counted divided by the incident flux. Thus, the product $N \sigma$ is equal to the area of the pixel times $\mathrm{QE}$, and is approximately $8 \times 10^{-18}$ $\mathrm{cm}^{2}$. This is significantly less than the cross section responsible for ghosting. ${ }^{6}$ This is also much smaller than the defect cross sections associated with deep optical defects in semiconductors $\left(10^{-16} \mathrm{~cm}^{2}\right)^{7}$

The activation energies associated with deep level defects in silicon are in a similar range of activation energies found in this study. ${ }^{8}$ The cross sections measured here, are, however, significantly smaller. It is well known that if light causes an electronic excitation of such deep level defects, the local configuration changes. These changes are often metastable. We propose a model where such photoinduced defects are defects that can respond "normally" to light in the sense that they have a reasonable cross section. The decay of these defects prior to being hit by a second photon precludes electron generation. Because the decay times are so short, the effective cross section appears much smaller than the computed one. This model precludes a proportional response to the incident irradiance and instead it should be proportional to the square of the irradiance. Preliminary data show that the response has a dependence on irradiance stronger than linear at $1570 \mathrm{~nm}$. This model seems to be at variance to what was described earlier: defects in the band gap that are the direct cause of the IR response. The complete model to explain all data is as follows. 
Below about $1330 \mathrm{~nm}$, defects in the gap are the main source of the response. Assuming that near band edge defects are the main source, then with an activation energy on the order of $0.2 \mathrm{eV}$ and a silicon band gap of $1.13 \mathrm{eV}$ (at $273 \mathrm{~K}$ ), only photons more than $0.93 \mathrm{eV}(<1330 \mathrm{~nm})$ are able to provide a direct signal. Impurities that have activation energies in the relevant range include: $\mathrm{In}, \mathrm{Sn}, \mathrm{C}, \mathrm{Cu}, \mathrm{Ti}, \mathrm{Au}, \mathrm{Zn}$, and $\mathrm{Ni}$. These are all likely impurities. The model for these shorter wavelengths means that there should be a linear relationship between intensity and response. We have measured this with the laser at $1310 \mathrm{~nm}$ and an approximate linear relationship is indeed found. Above $1330 \mathrm{~nm}$, an explanation involving metastable photoinduced defects is invoked to explain the extremely small cross sections.

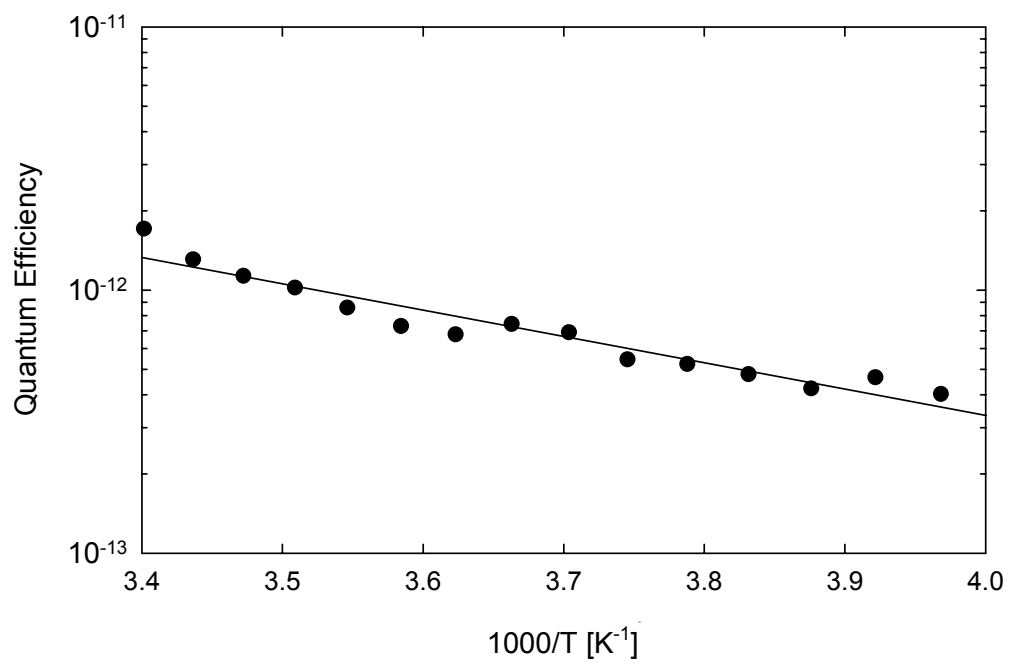

Figure 7. The average quantum efficiency as a function of the inverse temperature at $1570 \mathrm{~nm}$.

\section{CONCLUSIONS}

Silicon CCDs respond to infrared light. Electrons are excited to the conduction band, although the energy of the incoming light is smaller than the band gap. This signal is temperature dependent and hence, the quantum efficiency is temperature dependent. The QE depends exponentially on the inverse temperature and thus the QE can be characterized by an activation energy.

The activation energy was measured with an increasing uncertainty at the longer wavelengths. This uncertainty is quite possibly dependent on defects present in individual pixels and the difficulty in completely controlling the illumination. Nevertheless, a general trend is observable. The activation energies are in the range of $200 \mathrm{meV}$ and seem to decrease for the longer wavelengths.

The quantum efficiency also decreases with increasing wavelength. For the shortest measured wavelength $(1220 \mathrm{~nm})$ the QE is in the range of $10^{-7}$ and for the longest measured wavelength $(1580 \mathrm{~nm})$ it is in the range of $10^{-13}$. The QE decreases by a factor of $10^{-6}$. Compared to visible light with a QE close to one, these QEs are insignificant but are not equal to zero as normally is assumed.

Based on the measurements a cross section was calculated at $1570 \mathrm{~nm}$. This cross section is much smaller than expected. It is known that photoinduced defects are frequently metastable at room temperatures. The existence of the extremely small cross section can be explained by the fact that first a defect needs to be photoinduced and a second photon is needed to emit the electron into the conduction band. This is the model for the longer wavelengths. For wavelengths less than about $1330 \mathrm{~nm}$, defects in the band gap are the main source of the response. Potentially, this method might be used to probe the type of impurities present.

It is the long integration times possible in CCDs, which makes it possible to probe defects in semiconductors heretofore not easily accessible. 


\section{ACKNOWLEDGEMENTS}

Work supported by Technologiestichting STW. We thank Herman Peek, Albert Theuwissen, and Morley Blouke for fruitful discussions.

\section{REFERENCES:}

1 J.R. Janesick, Scientific Charge-Coupled Devices, 172, SPIE-The International Society for Optical Engineering, Bellingham, WA, 2001

2 A.J.P. Theuwissen, Solid-State Imaging with Charge-Coupled Devices, 298, Kluwer Academic Publishers, Amsterdam, 1996

3 I. Glass, Handbook of Infrared Astronomy, 142, Cambridge University Press, New York, 1999

4 D. E. Groom, S. E. Holland, M. E. Levi, N. P. Palaio, S. Perlmutter, R. J. Stover, and M. Wei, "Quantum Efficiency of a Back-illuminated CCD Imager: An Optical Approach," SPIE 3649, 80-90 (1999).

5 F.A. Jenkins and H.E. White, Fundamentals of Optics, 370, 4th edition, McGraw Hill, New York, 1981

6 A. Rest, L. Mündermann, R. Widenhorn, E. Bodegom, and T.C. McGlinn, "Residual images in charge-coupled device detectors," Rev. Scient. Instr. 73, 2028 (2002)

7 D. Redfield, and R. H. Bube, Photoinduced defects in semiconductors, 3, Cambridge University Press, New York, 1996

8 G.D. Watkins, “The Lattice Vacancy in Silicon,” Deep Centers in Semiconductors, ed. S.T. Pantelides, 177-214, Gordon and Breach Science Publishers, Philadelphia, 1992 